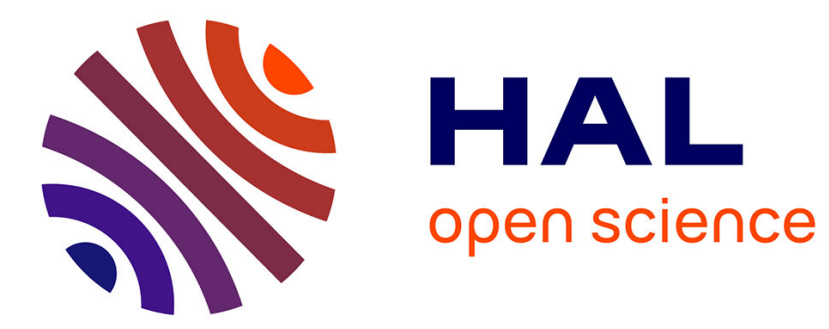

\title{
Les arts et les âges de la vie en Chine
}

\author{
Yolaine Escande
}

\section{To cite this version:}

Yolaine Escande. Les arts et les âges de la vie en Chine. Communications, 2021, Les arts et les âges de la vie, $n^{\circ} 109$ (2), pp.49-61. 10.3917/commu.109.0049 . hal-03387967

\section{HAL Id: hal-03387967 https://hal.science/hal-03387967}

Submitted on 28 Dec 2021

HAL is a multi-disciplinary open access archive for the deposit and dissemination of scientific research documents, whether they are published or not. The documents may come from teaching and research institutions in France or abroad, or from public or private research centers.
L'archive ouverte pluridisciplinaire HAL, est destinée au dépôt et à la diffusion de documents scientifiques de niveau recherche, publiés ou non, émanant des établissements d'enseignement et de recherche français ou étrangers, des laboratoires publics ou privés. 


\author{
Yolaine Escande \\ "Les arts et les âges de la vie en Chine », \\ Communications, 2021/2 (n 109), p. 49-61
}

En Chine, pratiquer une activité artistique est très valorisé, depuis les temps les plus anciens. Cela est dû au sens que les Chinois attribuent au terme «art», yi 藝, qui a pour étymologie «planter, cultiver», ce qui désigne la «culture de soi ». L'art pour les Chinois n'a donc rien à voir avec la science ou la technique, mais il est intimement lié à l'éducation, à la formation de l'homme de bien, depuis l'éloge qu'en a fait Confucius. Celui-ci exhorte, dans ses propos recueillis par ses disciples dans les Entretiens :

«Concentre ta volonté sur la Voie, prends appui sur la Vertu, modèle tes actions sur le ren, et prends ton plaisir dans les $\operatorname{arts}^{1}$.»

Les « arts » à l'époque de Confucius portaient sur les activités servant à la formation de l'homme de bien, à savoir les rites, la musique, l'écriture, le tir à l'arc, la conduite des chars, les mathématiques. Par la suite, ils ont désigné quatre activités, à l'exclusion de toutes les autres : la poésie, la musique, l'écriture, la peinture. Dans cette « culture de soi », l'apprentissage, en particulier dans l'enfance, est primordial. Si Confucius ne parle pas de jeu, il met néanmoins en avant le " plaisir », essentiel dans l'apprentissage. La tradition chinoise distingue très nettement l'enfance, l'âge adulte et la vieillesse. À ce propos, Confucius aurait également affirmé, dans les Entretiens :

«À quinze ans, je résolus d'apprendre. À trente ans, j'étais debout dans la voie. À quarante ans, je n'éprouvais plus aucun doute.

À cinquante ans, je connaissais le décret du ciel.

À soixante ans, j'avais une oreille parfaitement accordée.

À soixante-dix ans, j’agissais selon mon cœur, sans pour autant transgresser aucune règle ${ }^{2}$.»

La première phrase laisse entendre qu'avant quinze ans, on apprend par plaisir, c'està-dire sans avoir à se forcer, alors qu'à quinze ans, l'apprentissage est un choix. À 
trente ans, «debout dans la voie » signifie être suffisamment sûr de soi pour oser s'affirmer. C'est pourquoi à quarante ans, Confucius n'a plus de doute. Autrement dit, l'énergie vitale peut progresser jusqu'à quarante ans. À cinquante ans, « connaître le décret du ciel» signifie connaître ses limites, c'est aussi l'âge où l'énergie vitale commence à décliner. À soixante ans, « une oreille parfaitement accordée » veut dire que Confucius est complètement en accord avec lui-même, sans illusion et sans prétention non plus. À soixante-dix ans, agir selon son cœur sans transgresser de règle, c'est atteindre à la vraie liberté d'expression. Cela correspond, appliqué dans le domaine artistique, à l’idéal pour les Chinois.

Autrement dit, à chaque âge de la vie correspond une façon particulière de pratiquer les «arts », qui doit permettre d'éviter les défauts que pointe Confucius :

«Confucius dit: "L'homme de bien doit se prémunir contre trois maux : jeune, lorsque son sang et son souffle vital sont encore en effervescence, contre la débauche ; à l'âge mûr, lorsqu'ils sont en pleine vigueur, contre la pugnacité ; au soir de sa vie, lorsqu'ils se sont taris, contre la cupidité ${ }^{3} . "$ »

Toutes les étapes mentionnées plus haut par Confucius doivent en effet conduire à une expression artistique « sans contrainte », selon l'expression des textes théoriques sur l'art, c'est-à-dire sans effort volontaire et sans attente. C'est pourquoi les œuvres des artistes âgés sont toujours particulièrement appréciées : si elles manquent de fougue, elles expriment une liberté dans l'aisance, une maladresse au-delà de la technique, une forme d'honnêteté. Les gens âgés ne cherchent pas à plaire, ils n’ont plus rien à prouver, ils acquièrent donc la liberté. C'est pourquoi dans la tradition chinoise, les œuvres les plus valorisées d'un artiste sont généralement celles de la fin de sa vie, considérées comme nécessairement les plus abouties. Sur le marché de l'art, par exemple, les œuvres tardives du célèbre Zhang Daqian (Chang Dai-ch’ien, 18991983) sont actuellement de loin les plus recherchées et les plus onéreuses ${ }^{4}$. De même, les œuvres servant de modèles dans l'apprentissage de la calligraphie ou de la peinture aujourd'hui sont de préférence celles de la fin de la vie d'artistes reconnus (lorsque cela est possible bien entendu), comme par exemple l'Autel de l'immortelle Magu (Magu xiantan ji) datant de 771 (ill. 1) de Yan Zhenqing (709-785), en style régulier, ou $M a$ 
chaumière sur le mont Lu par Deng Shiru (1743-1805), datant de 1804 (ill. 2), en écriture sigillaire. En peinture, de toutes les œuvres de l'artiste, c'est le rouleau Habiter les monts Fuchun (Fuchun shanju tu) de Huang Gongwang (1269-1354), réalisé entre 1347 et 1350 (ill. 3), qui est préféré pour l'apprentissage des «montagnes et eaux»(shanshui), expression qui désigne le paysage pictural et littéraire.

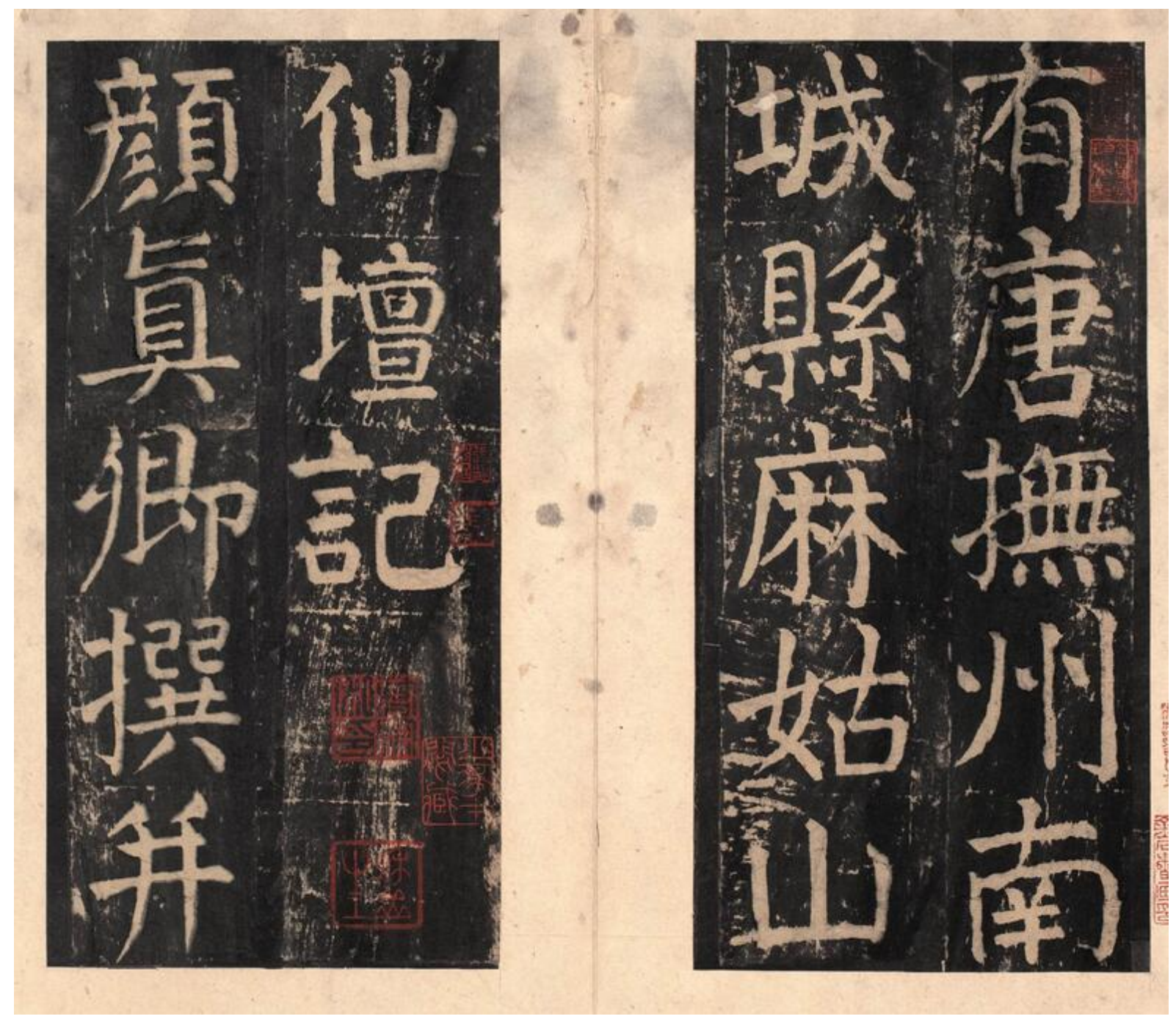

1. Yan Zhenqing (708-785), Annales de l'Immortelle Magu, 771, détail d'un estampage des Song, Pékin, musée de l'Ancien palais 


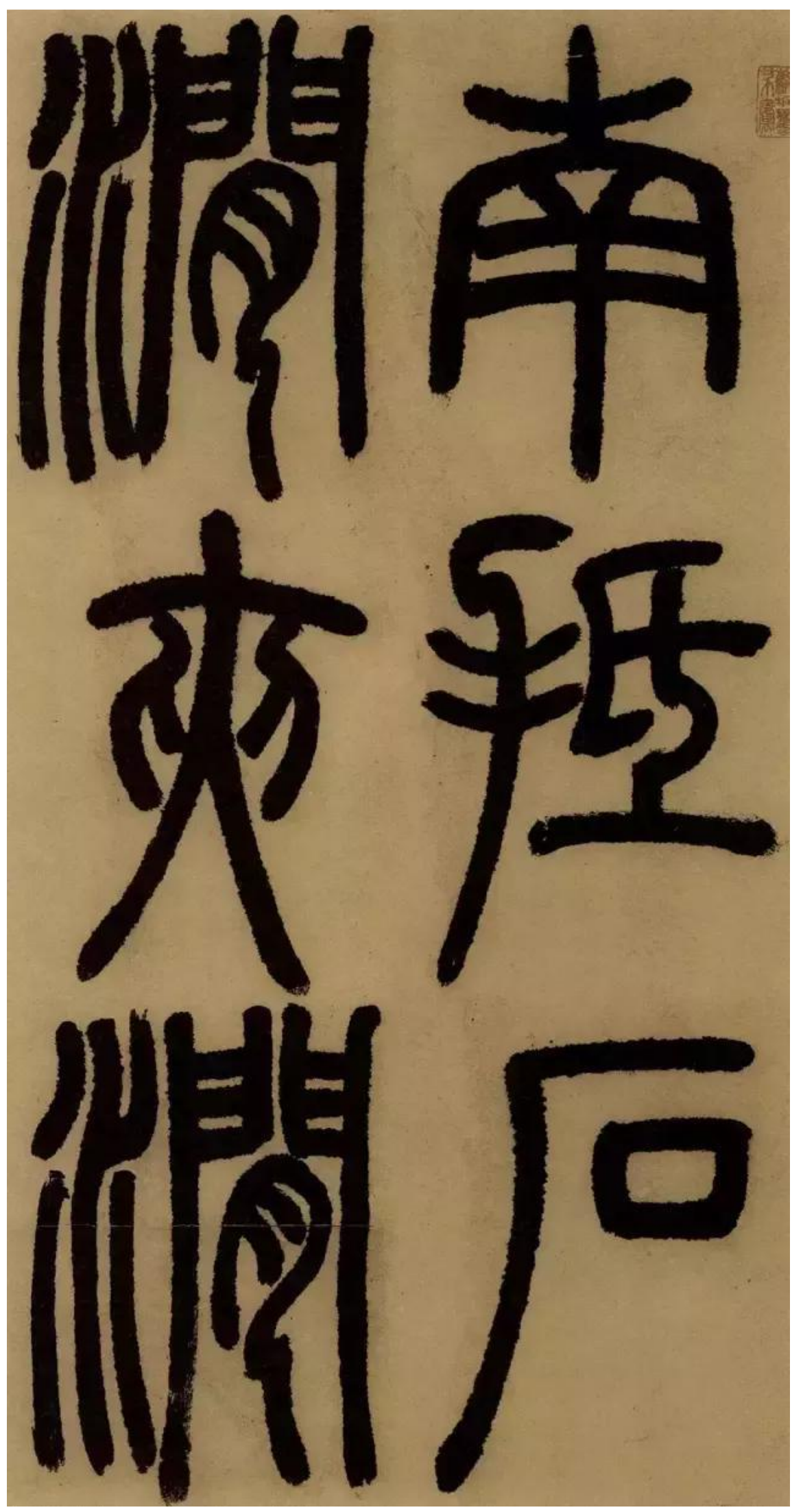

2. Deng Shiru (1743-1805), Ma chaumière sur le mont Lu, 1804, écriture sigillaire, détail, encre sur papier, Taipei, musée national du Palais

Escande Yolaine, «Les arts et les âges de la vie en Chine », Communications, 2021/2 (n 109), p. 49-61. DOI : 10.3917/commu.109.0049. URL : https://www.cairn.info/revue-communications2021-2-page-49.htm 


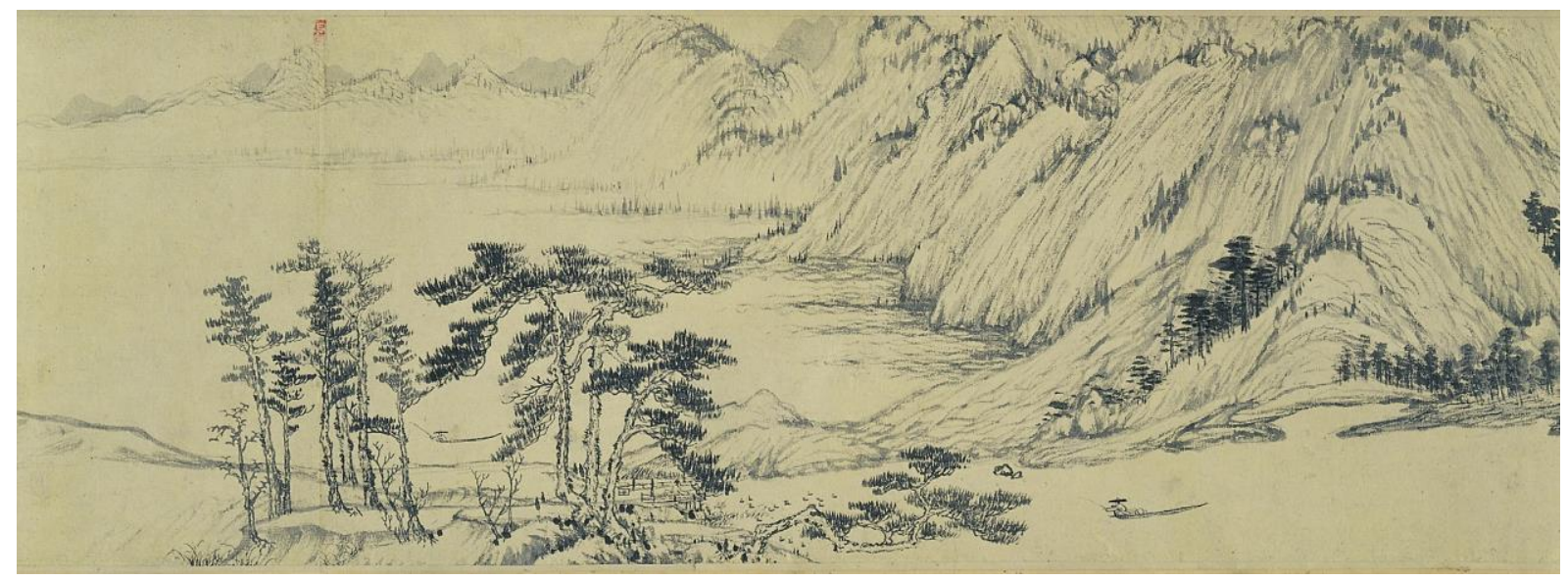

3. Huang Gongwang (1269-1354), Habiter les monts Fuchun, 1347-1350, détail, Taipei, musée national du Palais.

D'emblée, on peut retenir deux points essentiels : premièrement, l'art est un moyen de parfaire sa personnalité, au cours d'un processus qui dure toute la vie et que la vieillesse n'interrompt pas; deuxièmement, la vieillesse n'est pas considérée comme moins créatrice que l'âge mûr, bien au contraire, c'est à un âge avancé seulement que s'acquiert la liberté créatrice, considérée comme l'idéal suprême.

Le confucianisme a été le ciment de la société chinoise pendant près de trois mille ans, le courant principal de pensée. L'autre grand courant est le taoïsme, qui prône le retrait de la société pour se parfaire et devenir immortel. Même si, dans le taoïsme, la transmission est considérée comme impossible et si l'expérience créatrice reste à renouveler pour chaque personne ${ }^{5}$, néanmoins, les maittres taoïstes fondateurs de cette philosophie, Laozi et Zhuangzi, ont rédigé des traités censés transmettre leur enseignement. Au sein de ce paradoxe, les activités «artistiques » jouent un rôle fondamental, à commencer par l'art littéraire. Après tout, le nom de Laozi ne signifie-t-il pas « le vieil enfant»?

C’est pourquoi les artistes et théoriciens chinois se réfèrent au taoïsme, qui prône l'absence d'action, c'est-à-dire la création spontanée, sans volonté de créer, s'accordant au rythme de l'univers, à laquelle tendent naturellement les enfants d'une part et les artistes âgés d'autre part. 


\section{La pratique artistique en fonction de l'âge}

Selon les âges de la vie, la pratique artistique varie grandement en Chine. Par exemple, en calligraphie, on considère qu'il est plus facile de commencer, enfant, par réaliser de grands caractères, qui se tracent avec la totalité du corps. Ensuite seulement l'enfant prend-il le pinceau en main.

L'apprentissage passe par l'imitation de modèles. Le manuel d'instruction de base pour les enfants est un texte intitulé Mille caractères, dont aucun n'est répété. On apprend d'abord le style standard, celui de l'écriture imprimée aujourd'hui.

A l'adolescence, c'est-à-dire à partir du lycée, on réduit la taille des caractères et on apprend à tracer de petits caractères, ce qui est beaucoup plus difficile que les grands. On apprend aussi d'autres styles, en particulier le style semi-cursif, très utilisé dans l'écriture à la main, au stylo, au crayon ou au pinceau.

Dans l'apprentissage, on constate donc une nette séparation en Chine entre l'enfance d'un côté, et l'adolescence et l'âge adulte de l'autre.

A l'âge mûr, on doit savoir pratiquer tous les styles, plus ou moins, et on se met à la cursive, style qui exige une grande maitrise mais qui donne aussi beaucoup de liberté. L'apprentissage de la maitrise du pinceau et de la composition des caractères d'écriture permet d'acquérir une très grande sûreté du geste, des rapports des formes entre elles. Par exemple, dans les manga, dans les caricatures, cela peut être d'une grande aide.

Ensuite, à un âge avancé, en général, on commence à y voir moins bien. C'est pourquoi de nombreux calligraphes réalisent de grands caractères, voire des caractères monumentaux. La calligraphie des gens âgés est très appréciée, car elle retrouve une certaine maladresse, par manque de force, qui est compensée par la sûreté du geste et la maîtrise de la technique, en particulier la composition des caractères d'écriture.

En peinture, traditionnellement, l'apprentissage des peintres commence par la technique minutieuse ou détaillée, qui exige beaucoup de temps et de soin, et surtout de bons yeux (ill. 4). 


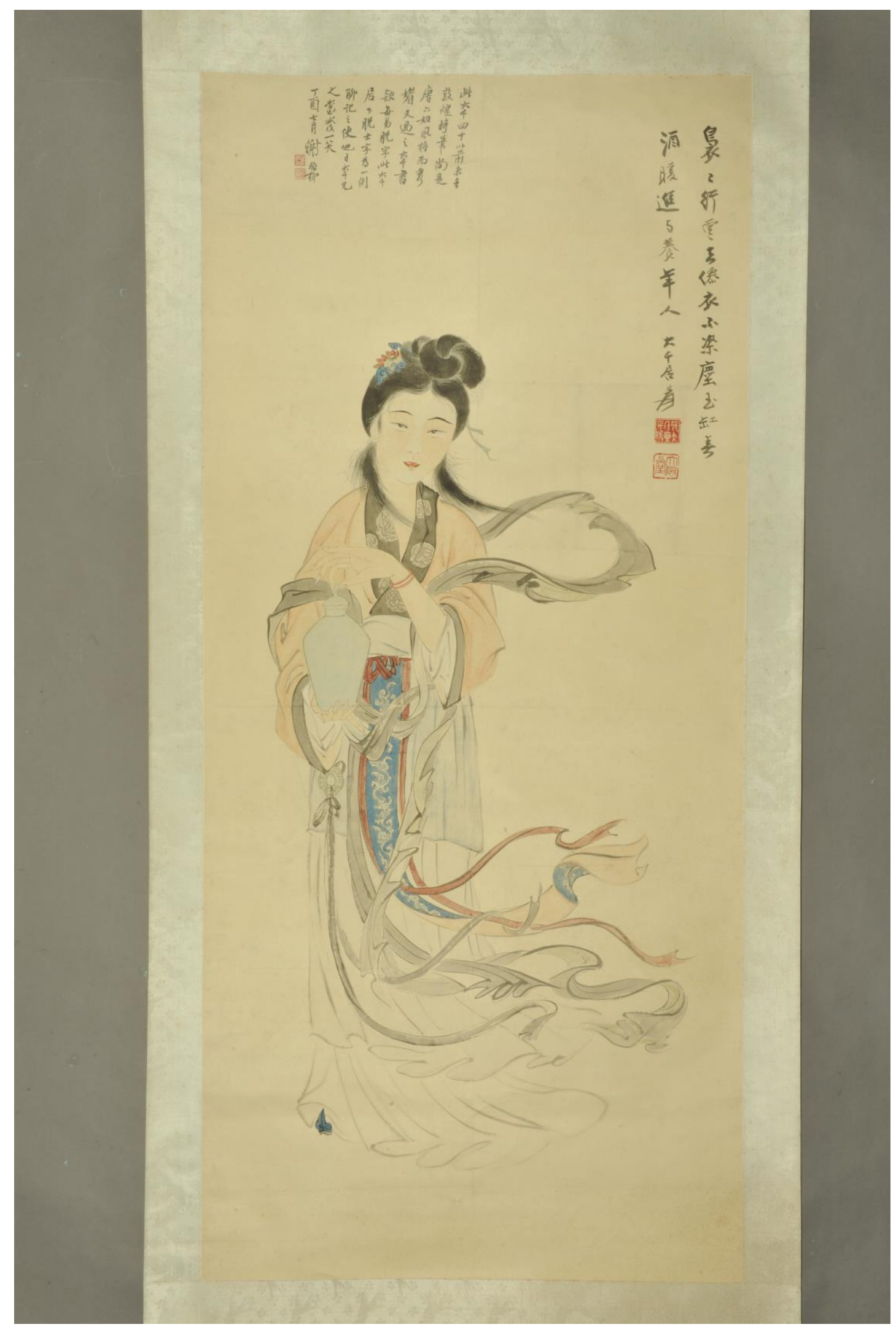

4. Zhang Daqian (1899-1983), Figure féminine, encre et couleurs sur papier, style minutieux, musée d'Art de Pékin. Avec l'aimable autorisation du musée des Beaux-Arts de Gaillac.

Escande Yolaine, «Les arts et les âges de la vie en Chine », Communications, 2021/2 (n 109), p. 49-61. DOI : 10.3917/commu.109.0049. URL : https://www.cairn.info/revue-communications2021-2-page-49.htm 
L'apprenti peintre commence par la maitrise de la technique du style méticuleux, littéralement « pinceau-travail » (gongbi) : par exemple, le peintre Gao Qipei (16601734) explique l'évolution de la pratique de son art dans une inscription sur une de ses œuvres, intitulée Regarder vers le haut et vers le bas:

«Quand j'étais jeune, j’aimais peindre en style méticuleux (gong) ${ }^{6}$. Il me fallait plusieurs mois pour achever une composition et, dès qu'elle était finie, je la laissais de côté sans la regarder. Plus tard, j'ai voulu pratiquer un tracé suggestif (xieyl). »

Après avoir acquis la maîtrise du pinceau minutieux, les peintres se consacrent, plus tard, souvent pour des raisons physiques, à la peinture suggestive : la peinture détaillée use les yeux et bannit tout tremblement. Alors que la peinture suggestive, réalisée rapidement, demande une grande maîtrise de la composition et du coup de pinceau, qui ne peut s'acquérir qu'avec l'expérience, mais tolère un appui du pinceau très marqué (ill. 5). 


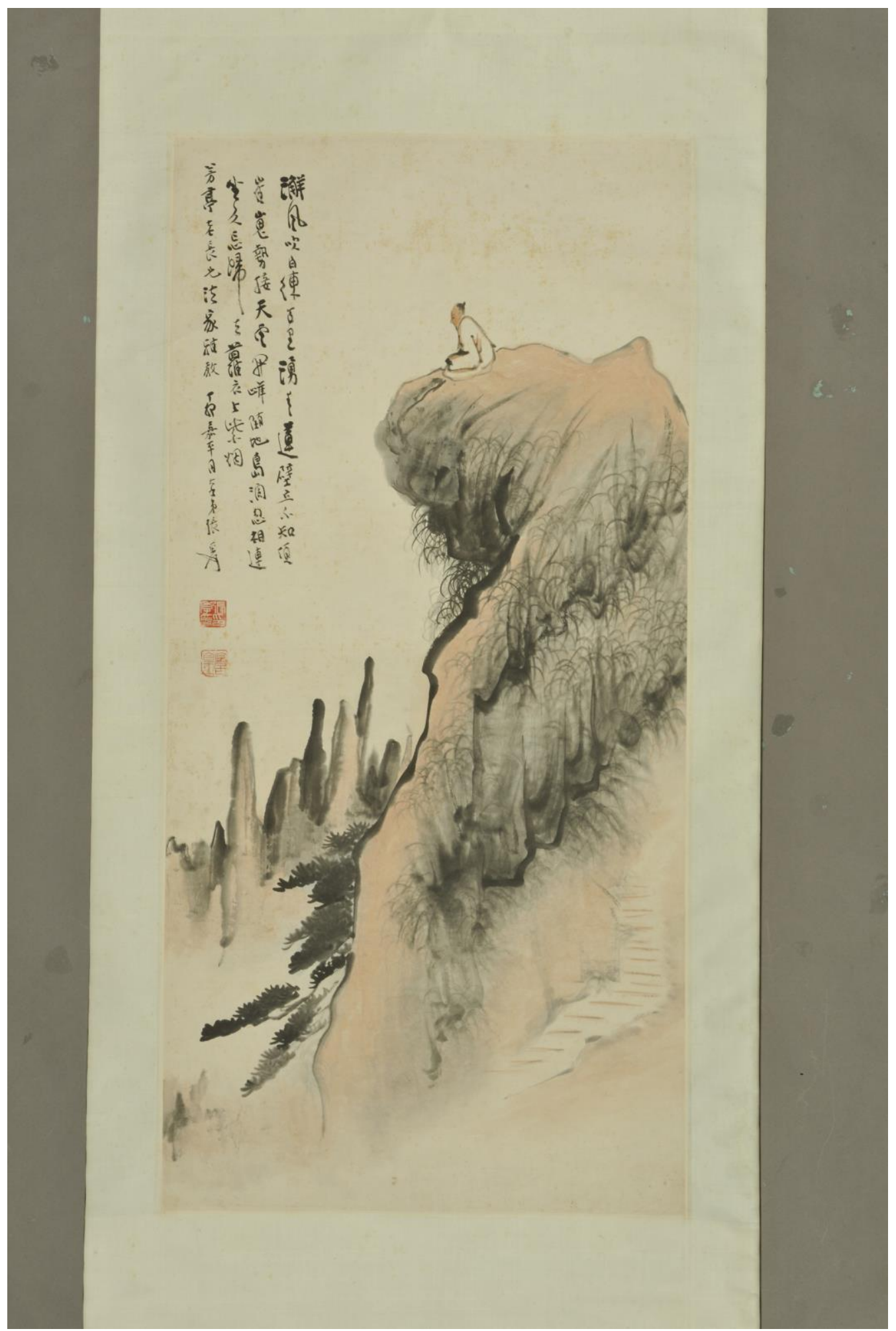

5. Zhang Daqian (1899-1983), Montagne, eau et personnage, encre sur papier, musée d’Art de Pékin. Avec l'aimable autorisation du musée des Beaux-Arts de Gaillac.

Gao Qipei oppose ainsi deux styles picturaux : méticuleux et suggestif. Le style méticuleux est souvent attribué aux professionnels et académiciens, aux artisans,

Escande Yolaine, «Les arts et les âges de la vie en Chine », Communications, $2021 / 2$ (n 109), p. 49-61. DOI : 10.3917/commu.109.0049. URL : https://www.cairn.info/revue-communications2021-2-page-49.htm 
alors que le style suggestif est celui des peintres d'un âge avancé. C'est également le style que revendiquent les lettrés (même s'ils peuvent aussi pratiquer le style minutieux occasionnellement), dont la théorie et la pratique les distinguent des professionnels et artisans. Par conséquent, la forme artistique la plus valorisée dans l'histoire chinoise, celle des lettrés, s'apparente à la pratique de personnes âgées. Il faut préciser que l'idéal pour tout Chinois est de parvenir à l'immortalité, or le grand âge est un premier pas vers cet idéal.

Inversement, la croyance veut que la pratique des arts soit la meilleure voie pour atteindre la longévité. Cette croyance, très ancrée et vivace de nos jours encore, veut que les personnes les plus admirables soient celles qui vivent longtemps car elles ont réussi à entretenir leur souffle vital et à s'approcher des qualités des immortels. Contrairement aux croyances de l'Egypte ancienne ou de l'Inde, la vie ici-bas n'est pas conçue comme la préparation pour une autre vie dans l'au-delà. C'est pourquoi le théoricien Zhou Xinglian (actif vers 1821-1850) affirme :

«Pratiquer la calligraphie permet de nourrir son souffle vital et même de le renforcer ${ }^{7}$.»

Et la peinture, en particulier de «montagnes et d'eaux» (shanshui), est censée prolonger la vie, aussi bien de ceux qui la pratiquent que de ceux qui l'admirent : le peintre et théoricien Wang Yu (1714-1748) des Qing explique ainsi :

«Dans l'étude de la peinture, on peut nourrir sa nature et ses sentiments, mais aussi se débarrasser de sa mauvaise humeur, rompre la solitude mélancolique, détendre son esprit sous pression, et accueillir les souffles de la tranquillité. Les anciens disaient que les peintres de shanshui jouissaient pour la plupart d'une grande longévité parce qu'ils étaient nourris des brumes et vapeurs et n'avaient sous les yeux que les ressorts de la vie. Puisque, depuis l'antiquité, la majorité des grands peintres de shanshui ont profité de leur longévité, ce dicton doit être vrai $^{8} . »$

Autrement dit, respirer les vapeurs cosmiques en étant «nourri de brumes et vapeurs » revient à être un immortel.

Cette croyance est étonnamment étayée par les récentes recherches en neuropsychologie de Henry Kao (Gao Shang-ren) ${ }^{9}$ sur l'écriture au pinceau ; les travaux de 
ce chercheur ne concernent pas la calligraphie en tant qu'art, mais se penchent sur son utilité dans le fonctionnement mécanique du corps humain. La calligraphie est perçue comme une thérapie : lorsqu'un patient écrit au pinceau, son souffle est plus régulier et profond et son cœur plus lent que lorsqu'il reste assis à lire par exemple. Par conséquent, sa pression artérielle baisse, etc. et l'écriture au pinceau contribue effectivement à sa longévité ${ }^{10}$.

Pour autant, l'estimation du grand âge ne signifie pas que les œuvres des enfants soient déconsidérées ou sans valeur. Le peintre Gu Ningyuan (actif fin du XVI siècle) décrit les œuvres des enfants, des femmes et des gens sans prétention en les qualifiant de «crues, naïves, fraiches» (sheng), de «maladroites» (zhuo), par opposition à celles des artistes accomplis, « habiles» (shou) et «techniquement achevées » (gong) :

«En peinture il faut rechercher la fraîcheur (sheng) après être parvenu à la dextérité (shou). [...] La perfection technique (gong) ne vaut pas la maladresse $(\text { zhuo })^{11} . »$

La fraîcheur ou « crudité » ne s’atteint que lorsque la technique est oubliée, elle n'est pas donnée a priori et demeure un idéal. Gu Ningyuan explique en effet :

«Les apprentis tombent dans les ornières dès qu'ils commencent. Mais les enfants, les femmes et les gens sans prétention qui peignent souvent pour leur propre plaisir, n’osent pas le montrer à autrui. Même si leurs œuvres ne sont pas vivantes, elles ont ce à quoi les artistes accomplis ne parviennent pas : la fraîcheur (sheng) et la maladresse (zhuo). Ces personnes disent que la fraîcheur et la naïveté n'ont rien à voir avec l'apprentissage des normes et que leur nature originelle n'est qu'en germe, comme les premières déterminations qui sont issues du chaos ; leurs œuvres ne sont que des ébauches ${ }^{12}$. »

Reste que ces ébauches sont admirables en ce qu'elles ne font montre d'aucune dextérité et qu'elles expriment l'aisance, ce à quoi ne parviennent que les gens âgés.

Dans l'évolution de l'apprentissage en art, jeunes et vieux présentent également des caractéristiques radicalement différentes, comme le pointe le calligraphe et théoricien Sun Guoting (648 ?-702 ?) des Tang :

«Pour la compréhension des lois et des principes, les jeunes ne valent pas les vieux. Mais dans l'apprentissage pratique des normes et règles, les vieux ne 
valent pas les jeunes. Pour la pensée, les vieux sont merveilleusement supérieurs ; pour l'étude [pratique], les jeunes ont plus d'ardeur. Ceux qui ne relâchent jamais leurs efforts parcourent trois phases, dont la maitrise de chacune permet le passage à la suivante. Au début de l'apprentissage, ils ne recherchent que l'équilibre et la régularité dans la structure et la composition. L'équilibre et la régularité atteints, [ils doivent] s'attacher à rechercher l'extrême hardiesse et, une fois celle-ci aboutie, [ils doivent] revenir à l'équilibre et à la régularité. Dans la première phase, ils sont en-deçà [de la technique] ; dans la deuxième, ils sont au-delà ; enfin [à la troisième phase], c'est la maitrise complète. Au stade de la maitrise complète, l'homme et son écriture ont ensemble atteint la maturité ${ }^{13}$.»

La pratique artistique sert donc dans un sens confucéen à atteindre la maturité, c'està-dire l'expression dans la sérénité. L'auteur oppose d'abord les jeunes et les vieux, avant de déterminer trois phases dans l'apprentissage : la formation technique, la maîtrise technique, le dépassement de la technique. Ces trois phases, en réalité connues depuis les temps les plus anciens, persistent dans l'apprentissage des arts, quels qu'ils soient.

Dans la pratique contemporaine, en peinture et calligraphie, en Chine, le moyen pour connaître son niveau d'avancement dans la maitrise de l'art est de passer par des concours-expositions ; ceux-ci s'adressent aux artistes en fonction de leur âge. Par exemple, il existe des concours régionaux, nationaux ou internationaux de jeunes calligraphes ou de jeunes peintres. Cela désigne les artistes débutants, qui ont moins de trente ans.

L'évaluation des œuvres pendant les concours de calligraphie et de peinture est assurée par des calligraphes et des peintres, déjà affirmés ou reconnus, réunis dans des comités de sélection. Pour participer à un concours de calligraphie ou de peinture, il faut absolument être «invité ». En d'autres termes, on ne peut pas se présenter en tant qu'individu, mais il faut être recommandé par un artiste. Pour cela, il faut absolument faire partie d'une association, quelle qu'elle soit. Cela peut être une association de quartier.

Pour participer à un premier concours de calligraphie ou de peinture, c'est évidemment le maître auprès duquel se déroule l’apprentissage qui recommande le 
ou la disciple. Le prestige de ce maître est très important. Il faut donc bien choisir son maître lorsqu'on apprend la calligraphie ou la peinture.

De ceci, un autre point essentiel peut être retenu : l'adolescence n'existe pas dans l'apprentissage artistique, le passage se fait directement de l'enfance à l'âge adulte.

\section{Les arts et les âges de la vie dans le cinéma}

La question porte cette fois non plus sur l'âge de celui ou celle qui pratique une activité artistique, mais sur la représentation des arts et des âges de la vie dans le cinéma chinois, et plus particulièrement dans celui des années 1990, à une époque où la Chine s'ouvre sur le monde depuis plus d'une décennie, où Hong Kong prépare la rétrocession à la Chine, et où Taïwan, alors jeune démocratie, s’interroge sur son identité. Le cinéma des années 1990 est déjà mondialisé, tout en précédant les productions ou co-productions hollywoodiennes en Chine. Les quatre films mentionnés mettent en scène un maître dans une activité artistique et son disciple, ou l'évolution d'un mâttre dans son art au cours de sa vie.

Les films servant de référence sont La vie sur mille cordes (Bianzou bianchang) de Chen Kaige (1991), Le Maître de marionnettes (Ximeng rensheng) de Hou Hsiao-hsien (1993), La Légende du dragon rouge (Hong Xiguan zhi Shaolin wuzu) de Wong Jing et Corey Yuen (1994, version internationale, 2002) et Le Roi des masques (Bianlian) de Wu Tianming (1996).

Le choix de ces films, au détriment d'autres, est motivé par le fait que l'enfance et la vieillesse dans l'apprentissage ou la pratique d'un art sont au centre de la réflexion. Les arts considérés sont la musique, le théâtre de marionnettes, les arts martiaux et le théâtre des masques. Les thèmes de la transmission, du passage de l'enfance à l'âge adulte, du grand âge, de la vie et de la mort, de l'immortalité, du rôle des arts sont également traités.

Le Maître de marionnettes rapporte l'histoire vraie du plus grand marionnettiste de son temps et mondialement célèbre, Li Tien-lu (1910-1998), à Taïwan, narrée par le vieil 
homme en voix-off ou face à la caméra. Li narre les principaux épisodes de son enfance et de sa vie d'adulte sous l'occupation japonaise de Taïwan (1895-1945). Le film se termine à la fin de la seconde guerre mondiale. Li Tien-lu apprend les rudiments de l'art de la manipulation des marionnettes à main sous la direction de son père. A quatorze ans, il donne sa première représentation théâtrale et fonde sa propre compagnie à vingt-deux ans.

L'enfance, difficile en raison de la mort de sa mère alors qu'il n'avait que neuf ans, est la période de l'apprentissage. Mais Li Tianlu passe directement de l'enfance à la vie d'adulte, dès lors qu'il donne ses propres représentations. Certes deux acteurs jouent le maitre enfant et adulte. Mais la présence à l'écran est celle, omniprésente, du vieux maître lui-même qui, par son ironie et son humour, transmet au spectateur une forme de légèreté, de distance malgré les drames qui ont alourdi sa vie (enfant, la perte de sa mère, adulte, la perte de son fils, la famine, la pression de l'occupant, etc.) et la foi en son art, qu'il n'a ensuite cessé de produire et d'enseigner. Par sa seule présence, son exemple et sa sérénité désabusée, Li Tien-lu, déjà une icône dans son pays, devient un symbole au plan international du destin de l'île. Les étapes de sa vie et de son art sont enfin autant de jalons et d'exemplifications des paroles de Confucius.

La Vie sur mille cordes, intitulé en chinois Marcher en chantant, met en scène deux musiciens aveugles, un vieux maitre et son tout jeune disciple, qui chantent des poèmes en s'accompagnant d'un luth, et racontent des histoires légendaires, se déplaçant de village en village. Dans le film, dans une Chine sans âge, le maître est qualifié de « saint », shensheng, ou shengren, parce qu'il parvient, par ses chants, à arrêter les conflits et guerres entre villageois. Un saint n'est pas un personnage canonisé par une Eglise, mais quelqu'un qui est parvenu, par son accomplissement intérieur, à l'union avec le monde et qui accepte de revenir parmi les hommes pour leur montrer le chemin vers la sagesse. C'est donc un modèle qui, par sa générosité et sa bienveillance, irradie sur les autres. Le film montre la transmission entre le maitre et son disciple, qui devient « saint » à son tour à la mort du maitre. 
La mort du maitre, sur laquelle commence le film, est une étape initiatique qui conduit le disciple à devenir maître à son tour. D'enfant ou de disciple, il passe au rôle et au statut de maître ou d'adulte. En d'autres termes, il ne peut plus se contenter de suivre, il faut qu'il se mette à créer lui aussi. La disparition du maître est à la fois une douleur, puisqu'il perd son repère, et une libération, puisqu'il peut créer à sa guise. Le film montre bien la différence des âges: le disciple chante faux, contrairement au maître, a du mal à se concentrer, etc. Alors que le maître est montré sans fard, avec ses rides, ses souffrances physiques, ses doutes, mais aussi son assurance artistique. Au jeune, les joies de la découverte des sens, de l'amour, du questionnement. Au vieux, celles de voir son disciple s'émanciper, mais aussi son inquiétude et sa crainte qu'il ne puisse pas faire face après sa disparition. Leurs rôles sont très différenciés et marqués. On a à la fois l'expression de la philosophie confucéenne qui prône de participer à la vie sociale pour améliorer la société, et celle de la philosophie taoïste qui met en avant le retrait de la vie sociale et qui dénie toute transmission. De fait, le maître ne transmet pas ce qu'il sait, mais ce qu'il est. L'important, ce n'est pas la technique, que tout le monde peut apprendre, mais le fait de se comporter en tant qu'être humain. C'est d'ailleurs le premier rôle de l'art en Chine, former des « hommes de bien ».

Dans Le Roi des masques, situé temporellement dans les années 1930, la problématique est assez proche : M. Wang (dont le patronyme signifie « roi ») est un vieil artiste itinérant sur les fleuves de la province centrale du Sichuan, vivant sur sa barque avec son singe Général. Il gagne sa vie en changeant en un éclair de masque (bianlian) sur son visage ${ }^{14}$, comme par magie ; mais arrivé à un âge avancé, il craint de disparaître sans avoir transmis son art à un héritier mâle, ainsi que le veut la tradition. Il se résout à acheter un petit-fils sur le marché des enfants, auquel il enseigne son art. Mais patatras, il s'avère que cet enfant n'est qu'une fille ! Après moult péripéties, le roi des masques finit par accepter de transmettre le secret de son art à la fillette. Ici ne sont présentés que deux âges de la vie : l'enfance et la vieillesse. L'art est ce qui permet de vivre, et c'est par conséquent un bien précieux, d'autant que l'époque est 
politiquement chaotique et socialement dure. La bonté humaine est ce qui permet à la fillette de se révolter contre le destin et de renverser les normes patriarcales, et au vieil artiste de bousculer ses représentations. La transmission, en outre, est primordiale, elle fait accéder le roi des masques à l'immortalité.

Dans la réalité, cet art traditionnel du Sichuan considéré comme un trésor national a été transmis à huit jeunes femmes dès 1998 par maître Peng Denghuai, ce qui provoqua un scandale ${ }^{15}$. Par ailleurs, depuis 2007, les femmes ont officiellement le droit d'apprendre cet art et de l'interpréter publiquement. Le film bouscule donc un tabou et provoque le spectateur, le conduisant à s'interroger sur les questions de légitimité de la transmission. Ce film, comme les exemples qui précèdent, se situe dans la croyance que l'art a le pouvoir de changer les gens, qu'il a une mission éducative, didactique et édifiante, tout comme la peinture à ses origines. Les deux âges de la vie, enfance et vieillesse se rejoignent en ce qu'ils ne sont pas piégés par les apparences, malgré les masques.

Enfin, La Légende du dragon rouge est un film de kung-fu dans la pure tradition hongkongaise. Les arts martiaux, contrairement au théâtre ou à l'opéra chinois, sont ouverts aux femmes depuis longtemps. Le film présente un père, moine de Shaolin, qui apprend les arts martiaux à son fils ; au cours de l'histoire, ils sont aidés par une petite voleuse et sa mère, elles aussi pratiquantes des arts martiaux, dans leur combat inégal contre l'oppresseur mandchou. L'action se passe en effet sous la dernière dynastie non-chinoise, celle des Mandchous. Les âges de la vie principaux dans le film sont l'enfance et la force de l'âge. L'action du film est rapide, les scènes de combat sont mises en avant et montrent la virtuosité du père qui défait à lui seul $\mathrm{x}$ combattants à la fois. Cependant, le fils lui vole souvent la vedette et fait montre de tout autant de virtuosité. La violence de l'action est atténuée par l'humour et la dérision. Nous sommes là dans la phase « maitrise » et « virtuosité » de l'art, et non dans la phase « expression créatrice ». Le père doit se battre sur tous les fronts et faire montre de sa puissance et de son habileté au combat, secondé de son fils, alors que dans d'autres films d'arts martiaux mettant en scène un vieux maître, celui-ci n'a 
même plus besoin de combattre pour défaire ses adversaires. C'est le cas notamment de Zatoichi, un vieil aveugle, dont les gestes sont si éprouvés qu'on ne les voit même pas (Zatoichi, 2003), exactement comme ceux du roi des masques, qui semblent tenir de la magie!

Pour résumer, et en guise de conclusion, s'il existe bien une façon particulière de pratiquer les arts en Chine en fonction de chaque âge de la vie, il apparaît nettement que l'âge le plus propice à la création artistique est la vieillesse. Au lieu de penser la dynamique créatrice et esthétique sur le modèle du cycle organique, et d'imaginer que la vieillesse correspond à un déclin de la création artistique et de l'expérience esthétique, au contraire, la vieillesse étant la marque de la longévité, et le premier stade vers l'immortalité, elle est considérée comme l'âge de la pleine liberté créatrice, de la présence au monde la plus entière et la plus intègre et de la capacité d'expression hors de toute contrainte sociale ou morale. Si les limites physiques sont bien présentes, en raison de l'affaiblissement des forces et de la dégénérescence des sens, de la vue en particulier, celles-ci ne sont pas considérées comme un obstacle, bien au contraire, puisqu'elles sont censées exprimer l'honnêteté à travers la maladresse non feinte.

\section{Yolaine Escande}

Directrice de recherche au CNRS

Centre de recherches sur les arts et le langage (CRAL, UMR 8566)

EHESS Paris

yolesc@ehess.fr 
Liste des illustrations

1. Yan Zhenqing (708-785), Annales de l'Immortelle Magu, 771, détail d'un estampage des Song, Pékin, musée de l'Ancien palais.

2. Deng Shiru (1743-1805), Ma chaumière sur le mont Lu, 1804, écriture sigillaire, détail, encre sur papier, Taipei, musée national du Palais.

3. Huang Gongwang (1269-1354), Habiter les monts Fuchun, 1347-1350, détail, Taipei, musée national du Palais.

4. Zhang Daqian (1899-1983), Figure féminine, encre et couleurs sur papier, style minutieux, musée d'Art de Pékin.

5. Zhang Daqian (1899-1983), Montagne, eau et personnage, encre sur papier, musée d'Art de Pékin.

Résumé :

L'article vise à montrer que, dans les arts chinois traditionnels et d'aujourd'hui, l'enfance et la vieillesse sont les deux âges les plus valorisés, et en particulier la vieillesse. Dans un premier temps, sont abordés les arts et les âges de la vie dans la peinture et la calligraphie chinoises, à travers l'âge de celui ou celle qui pratique une activité artistique, à partir de la confrontation de textes et de l'expérience artistique. Dans un second temps, est examinée la représentation des arts et des âges de la vie dans le cinéma chinois des années 1990 mettant en scène un maître et son disciple.

Mots-clés : calligraphie chinoise, peinture chinoise, vieillesse, enfance, cinéma chinois des années 1990

\section{Abstract :}

The paper aims to show that, in the Chinese traditional arts, and today, youth and old age are the most highly valued, especially old age. In the first part of the paper, 
the arts and the stages of life are discussed through Chinese painting and calligraphy: the age of the one who practices an artistic activity is examined, starting from setting together texts and artistic experience. In the second part, the representation of the arts and the stages of life are studied in 1990's Chinese movies in which master and disciple are put on the stage.

Keywords : Chinese calligraphy, Chinese painting, old age, youth, 1990's Chinese movies

\footnotetext{
${ }^{1}$ Trad. Anne Cheng, Entretiens de Confucius, Paris, Seuil, 1981, VII.6, p. 62.

${ }^{2}$ Ibidem II.4, p. 33.

${ }^{3}$ Ibidem XVI.7, p. 131.
}

${ }^{4}$ Zhang Daqian est certainement le plus célèbre artiste chinois du XX $\mathrm{XX}^{\mathrm{e}}$ siècle. Voir Pierre Haski, «Connaissez-vous Zhang Daqian, le Chinois qui a détrôné Picasso ? ", L'Obs, Rue 89, 3 mars 2012, article en ligne : https://www.nouvelobs.com/rue89/rue89-

chine/20120303.RUE8267/connaissez-vous-zhang-daqian-le-chinois-qui-a-detrone-picasso.html ; Liz Hammer, "Zhang Daqian. A Guide to China's Most Popular Artist », Christie's, 19 février 2019, article en ligne : https://www.christies.com/features/10-things-to-know-about-ZhangDaqian-9229-3.aspx .

${ }^{5}$ Traités chinois de peinture et de calligraphie, Tome 1, Paris, Klincksieck, 2003, p. 37-39.

${ }^{6}$ Le mot gong signifie « travail » et désigne les pratiques artisanales.

${ }^{7}$ Mon bumble opinion sur l'exercice de la calligraphie (Linchi guan jian), Huang Jian (éd.), Anthologie des traités sur la calligraphie des dynasties successives (Lidai shufa lunwen xuan), Shanghai, Shuhua chubanshe, 1979, p. 730.

${ }^{8}$ Propos de Dongzhuang sur la peinture (Dongzhuang lun hua, vers 1730), Yu Jianhua (éd.), Traités chinois sur la peinture par catégories (Zhongguo Hualun leibian), Pékin, Renmin meishu chubanshe, 1977, p. 187.

${ }^{9}$ Henry Kao (Gao Shang-ren), The Psychology of Calligraphy (Shufa xinlixue), Taipei, Dongda tushu, 1986.

${ }^{10}$ On peut lire en anglais : Henry S.R. Kao, "Shufa: Chinese calligraphic handwriting (CCH) for health and behavioural therapy", International Journal of Psychology Special Issue on the Indigenous Psychologies, Carl Martin Allwood, John W. Berry (éds.), août 2006, vol. 41, nº 4, p. 282-286. http://onlinelibrary.wiley.com/doi/10.1080/00207590544000059/abstract; Min Xu, Henry S. R. Kao, Manlin Zhang, Stewart P. W. Lam, Wei Wang, "Cognitive-Neural Effects of Brush Writing of Chinese Characters: Cortical Excitation of Theta Rhythm", Evidence-Based Complementary and Alternative Medicine, vol. 2013, Article ID 975190, 11 pages ; doi:10.1155/2013/975190, http://www.hindawi.com/journals/ecam/2013/975190/ ; Timothy CY Kwok, Xue Bai, Henry SR Kao, Jessie CY Li, Florence KY Ho " "Cognitive effects of calligraphy therapy for older people: a randomized controlled trial in Hong Kong", Clinical Interventions in Aging, 2011, n 6, p. 269-273. http://www.ncbi.nlm.nih.gov/pmc/articles/PMC3212418/

${ }^{11}$ Instructions sur la peinture (Huayin, vers 1570), in Yu Jianhua (éd.), Op. cit., p. 119.

${ }^{12}$ Ibidem.

${ }^{13}$ Traités chinois de peinture et de calligraphie, Tome 2, Paris, Klincksieck, 2010, p. 125-126. 
14 Sur cet art, voir Phyllis Xu, Maxim Dunca, «Chinese opera face changing : it's a kind of magic », Reuters, 11 décembre 2009, article en ligne : https://www.reuters.com/article/us-artchina-facechaning/chinese-opera-face-changing-its-a-kind-of-magic-idUSTRE5BA0I720091211 15 Voir Craig S. Smith, «Chengdu Journal; Man of a Thousand Faces Planning to Share a Few », The New York Times, 7 février 2001, article en ligne :

https://www.nytimes.com/2001/02/07/world/chengdu-journal-man-of-a-thousand-facesplanning-to-share-a-few.html 